\title{
Gluon condensate and the Polyakov loop
}

\author{
Juan Pablo Carlomagno ${ }^{1,2,3, \star}$ and Juan Cristóbal Rojas ${ }^{4, \star \star}$ \\ ${ }^{1}$ IFLP, CONICET - Dpto. de Física, Universidad Nacional de La Plata, C.C. 67, 1900 La Plata, Argentina \\ ${ }^{2}$ CONICET, Rivadavia 1917, 1033 Buenos Aires, Argentina \\ ${ }^{3}$ CONICET, Rivadavia 1917, 1033 Buenos Aires, Argentina \\ ${ }^{4}$ Departamento de Física, Universidad Católica del Norte, Angamos 0610, Antofagasta, Chile
}

\begin{abstract}
The temperature dependence of the gluon condensate is deduced from the Polyakov loop effective potential. It is shown that this approach provides a simple picture for the electric gluon condensate around the deconfinement temperature, showing that it drops to zero in a temperature range which is in good agreement with different pure gauge lattice results.
\end{abstract}

\section{Introduction}

One of the main aspects of quantum Chromodynamics (QCD) is the appearance of nontrivial gluon and chiral condensates.

A fundamental feature of QCD, regarded as a classical theory, is the fact that the trace of the energy-momentum tensor is zero, but, when it is considered as a full quantum theory, we have that such trace becomes non-zero, since there is an anomaly, implying a nonzero gluon condensate [1,2].

It is well established from Quantum Field Theory at Finite Temperature that, in presence of a thermal bath, the energy-momentum tensor can be split in a zero temperature part and a finite temperature contribution. The zero temperature contains the ultraviolet terms which give the anomalous term. On the other side, the finite temperature part is connected to the thermodynamic contribution to the energy density.

In this work, we compute the temperature dependence of the gluon condensate from the effective potential of a pure gauge theory. Our hypothesis is that the effective potential of self-interacting gluons comes from the Polyakov Loop potential.

\section{Polyakov Loop}

The order parameter which describes the deconfinement phase transition is the Polyakov loop [3, 4]

$$
\Phi(x)=\frac{1}{N_{c}} \operatorname{Tr} \mathcal{P} \exp \left(i \int_{0}^{1 / T} d x_{4} A_{4}\right) .
$$

\footnotetext{
^e-mail: carlomagno@fisica.unlp.edu.ar

$\star \star$ e-mail: jurojas@ucn.cl
} 
The center symmetry plays a key role since the Polyakov loop is not gauge invariant under transformations which belong to the gauge center. So, if the have $\Phi=0$, it means that there is a manifest $Z(N)$ symmetry, meaning that the system is confined. On the other side, if $\Phi \neq 0$, it means that the symmetry is broken and the system is deconfined.

Gauge freedom allow us to rotate the gauge field $A_{4}$ in order that it becomes static and diagonal. In that gauge fixing, the parameter $\Phi$, becomes the diagonal matrix $\exp \left(\mathrm{i} A_{4} / T\right)$, with $A_{4}=i A_{0}=$ $\operatorname{ig} \delta_{\mu 0} A_{a}^{\mu} \lambda^{a} / 2$, where $A_{a}^{\mu}$ are the $S U(3)$ color gauge fields. The traced Polyakov loop (PL) is given by $\Phi=\frac{1}{3} \operatorname{Tr} \exp \left(i A_{4} / T\right)$.

We work in the Polyakov gauge, in which the matrix $\Phi=\phi_{3} \lambda_{3}+\phi_{8} \lambda_{8}$ is diagonal [5]. The charge conjugation properties of the QCD Lagrangian imply that the mean field traced Polyakov loop field must be a real quantity [6]. Assuming that $\phi_{3}$ and $\phi_{8}$ are real-valued, $\phi_{8}$ has to be zero [7], and therefore

$$
\Phi=\frac{1}{3}\left[1+2 \cos \left(\phi_{3} / T\right)\right] .
$$

There is no unique choice for the the effective potential that describes the effective gauge field self-interactions. The Ansatz used come from the properties of pure gauge QCD. For example, it can be inspired by the logarithm of the Haar measure, the Jacobian which comes from the $S U(3)$ integration $[7,8]$

$$
\frac{\mathcal{U}_{\log }(\Phi, T)}{T^{4}}=-\frac{1}{2} a(T) \Phi^{2}+b(T) \log \left(1-6 \Phi^{2}+8 \Phi^{3}-3 \Phi^{4}\right) .
$$

Another possibility is basically a Ginzburg-Landau Ansatz [9, 10]

$$
\frac{\mathcal{U}_{\text {poly }}(\Phi, T)}{T^{4}}=-\frac{b_{2}(T)}{2} \Phi^{2}-\frac{b_{3}}{3} \Phi^{3}+\frac{b_{4}}{4} \Phi^{4} .
$$

There is also a mixed model proposed by Fukushima [11, 12]

$$
\mathcal{U}_{\text {Fuku }}(\Phi, T)=-b T\left[54 \exp (-a / T) \Phi^{2}+\log \left(1-6 \Phi^{2}+8 \Phi^{3}-3 \Phi^{4}\right)\right] .
$$

The coefficients $a_{i}(T), b_{i}(t)$ are obtained by fitting to the pure lattice QCD data and imposing physical conditions. For details see references given above.

\section{Thermal Gluon Condensate}

The pure gauge QCD Lagrangian is given by

$$
\mathcal{L}=-\frac{1}{4 g_{s}^{2}} G_{\mu \nu}^{a} G^{a \mu \nu},
$$

where

$$
G_{\mu \nu}^{a}=\partial_{\mu} A_{v}^{a}-\partial_{\nu} A_{\mu}^{a}-f_{b c}^{a} A_{\mu}^{b} A_{\nu}^{c} .
$$

We follow [13], where is proposed the following decomposition

$$
\mathcal{Z}=\int[d \tilde{A}] \exp \left\{\bigvee \mathcal{L}_{e f f}\right\} \approx \int[d \tilde{A}] \exp \left\{\frac{-1}{16 \pi \alpha_{s}(T)} \sum G_{\mu \nu}^{a} G^{a \mu \nu}\right\} .
$$

On the other side, we have the effective lagrangian

$$
\mathcal{U}=-T \ln \mathcal{Z}
$$




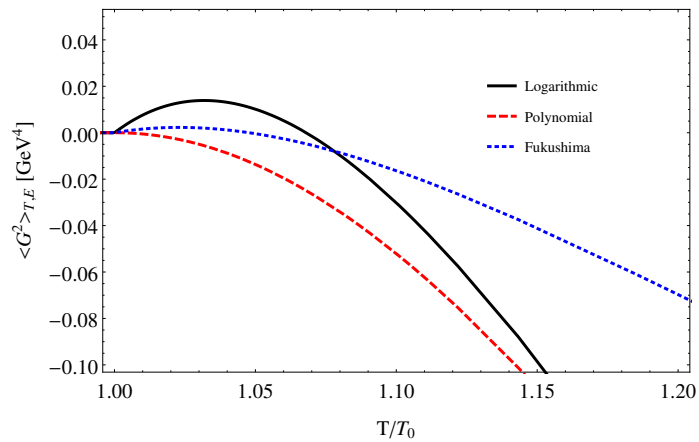

A direct comparison lead us to

$$
\left\langle G_{\mu \nu}^{a} G^{a \mu \nu}\right\rangle_{T}=16 \pi \frac{\partial \mathcal{U}}{\partial \alpha_{s}^{-1}} .
$$

The gluon tensor can be decomposed in an "electric" and "magnetic" terms:

$$
E_{i}=G_{i 4}, B_{k}=\frac{1}{2} \varepsilon_{i j k} G_{i j} \quad \rightarrow\left\langle\frac{\alpha_{s}}{\pi} G_{\mu \nu}^{a} G^{a \mu \nu}\right\rangle=\left\langle\frac{\alpha_{s}}{\pi} G_{\mu \nu}^{a} G^{a \mu \nu}\right\rangle_{E}+\left\langle\frac{\alpha_{s}}{\pi} G_{\mu \nu}^{a} G^{a \mu \nu}\right\rangle_{B}
$$

From lattice results [14], it can be realized that the magnetic part is not affected by temperature, then we can decompose the gluon condensate as

$$
\left\langle\frac{\alpha_{s}}{\pi} G_{\mu \nu}^{a} G^{a \mu \nu}\right\rangle \equiv\left\langle G^{2}\right\rangle=\left\langle G^{2}\right\rangle_{0}+\left\langle G^{2}\right\rangle_{T, E},
$$

from (10) we have

$$
\left\langle G^{2}\right\rangle_{T, E}=-\frac{4}{\pi} \alpha_{s}(T)^{2}\left(\frac{\partial \alpha_{s}(T)}{\partial T}\right)^{-1} \frac{\partial \mathcal{U}(\Phi, T)}{\partial T} .
$$

It remains to determine the value of $\alpha(T)$. If we assume that the temperature corresponds to the energy scale of the system, which is the natural criteria near $T_{0}$, the critical temperature. We choose two parametrizations for $\alpha$ which come from Schwinger-Dyson Analysis, given in [15],

$$
\alpha_{s}^{(1)}(T)=\frac{1}{1.16+T^{4} / \Lambda_{1}^{4}}\left[3.49\left(1.16-0.07\left(\frac{T^{2}}{\Lambda_{1}^{2}}\right)^{\frac{2}{3}}\right)+\left(\frac{T^{2}}{\Lambda_{1}^{2}}+2\right) \frac{T^{4}}{\Lambda_{1}^{4}} \alpha_{s}^{\beta_{1}}\right],
$$

where

$$
\alpha_{s}^{\beta_{1}}=\frac{4 \pi}{11 \ln \left(T^{2} / \Lambda_{1}^{2}\right)}\left[1-\frac{102}{11^{2}} \frac{\ln \left(T^{2} / \Lambda_{1}^{2}\right)}{T^{2} / \Lambda_{1}^{2}}\right] .
$$

And

$$
\alpha_{s}^{(2)}(T)=\frac{1}{15+T^{2} / \Lambda_{2}^{2}}\left[15 \times 2.6+\frac{4 \pi}{11}\left(\frac{1}{\ln \left(T^{2} / \Lambda_{2}^{2}\right)}-\frac{1}{T^{2} / \Lambda_{2}^{2}-1}\right) \frac{T^{2}}{\Lambda_{2}^{2}}\right]
$$

with $\Lambda_{2}=0.33 \mathrm{GeV}$. 


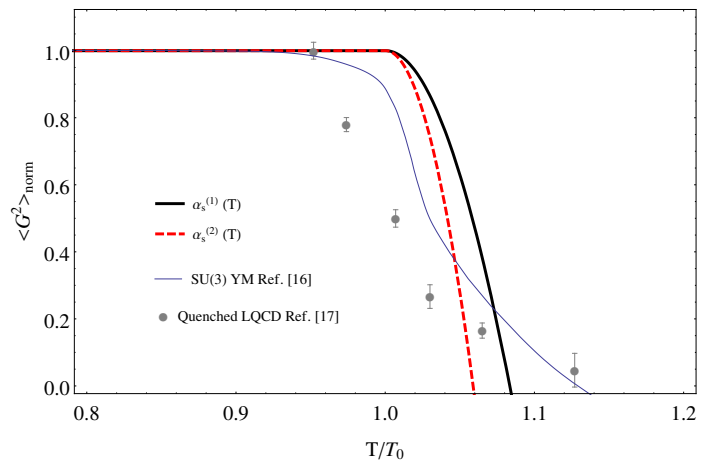

Figure 2. Normalized electric gluon condensate as a function of the reduced temperature for the polynomial potential and both strong coupling constants.

\section{Results and Conclusions}

Now is possible to plot the behavior of $\left\langle G^{2}\right\rangle_{T, E}$ using the different potentials (3), (4) and (5), those are depicted in Figure (1). First, it must be noticed that deconfinement occurs above the critical temperature $T_{0}$, implying that the effective potential must be zero below $T_{0}$. There we find that the best fit is given by the polynomial effective potential, since the Fukushima potential has an unstable behavior.

We have analyzed the temperature dependence of the gluon condensate for the three most used PL potentials in the literature, and proposed two parametrizations for the temperature dependence of the strong coupling constant. We showed that the polynomial effective potential has the best thermal behavior, while the qualitative dependence for both of the strong coupling parametrizations is equivalent. Finally, we conclude saying that the predictions obtained within this approach are in better agreement with estimates from lattice QCD than other more complex effective theories, and provides an accurate simple picture for the description of the electric gluon condensate around the deconfinement critical temperature.

\section{References}

[1] C. G. Callan, Jr., S. R. Coleman and R. Jackiw, Annals Phys. 59, 42 (1970)

[2] H. Leutwyler, "Deconfinement and Chiral Symmetry in QCD 20 Years Later", Vol. 2, P. M. Zerwas and H. A. Kastrup (Eds.), World Scientific, Singapore, 1993, pp. 693-716

[3] G. 't Hooft, Nucl. Phys. B 138, 1 (1978)

[4] A. M. Polyakov, Phys. Lett. 72B, 477 (1978)

[5] D. Diakonov and M. Oswald, Phys. Rev. D 70, 105016 (2004)

[6] A. Dumitru, R. D. Pisarski and D. Zschiesche, Phys. Rev. D 72, 065008 (2005)

[7] S. Roessner, C. Ratti and W. Weise, Phys. Rev. D 75, 034007 (2007)

[8] B. J. Schaefer, J. M. Pawlowski and J. Wambach, Phys. Rev. D 76, 074023 (2007)

[9] O. Scavenius, A. Dumitru and J. T. Lenaghan, Phys. Rev. C 66, 034903 (2002)

[10] C. Ratti, M. A. Thaler and W. Weise, Phys. Rev. D 73, 014019 (2006)

[11] K. Fukushima, Phys. Lett. B 591, 277 (2004)

[12] K. Fukushima, Phys. Rev. D 77, 114028 (2008) Erratum: [Phys. Rev. D 78, 039902 (2008)]

[13] N. O. Agasian, JETP Lett. 104 no.2, 71 (2016)

[14] M. D’Elia, A. Di Giacomo and E. Meggiolaro, Phys. Rev. D 67, 114504 (2003)

[15] A. Deur, S. J. Brodsky and G. F. de Teramond, Prog. Part. Nucl. Phys. 90, 1 (2016) 